\title{
Remoção cirúrgica de dente supranumerário incluso em região de mandíbula: relato de caso clínico
}

Surgical removal of supernumerary tooth included in the mandible region: clinical case report

Extirpación quirúrgica de diente supernumerario incluido en la región mandibular: reporte de caso clínico

Ygor Alexandre Beserra DE SOUSA

Departamento de Odontologia, Universidade Estadual da Paraíba (UEPB) 58429-500 Campina Grande - PB, Brasil https://orcid.org/0000-0002-0690-521X

Carlus Alberto Oliveira DOS SANTOS

Programa de Pós-Graduação em Odontologia, Universidade Estadual da Paraíba (UEPB) 58429-500 Campina Grande - PB, Brasil https://orcid.org/0000-0002-5988-1186

Ana Karina Almeida ROLIM

Programa de Pós-Graduação em Odontologia, Universidade Estadual da Paraíba (UEPB) 58429-500 Campina Grande - PB, Brasil https://orcid.org/0000-0001-6910-2898

Jussara da Silva BARBOSA

Programa de Pós-Graduação em Odontologia, Universidade Estadual da Paraíba (UEPB) 58429-500 Campina Grande - PB, Brasil https://orcid.org/0000-0001-6123-5266

Diego Filipe BEZERRA SILVA

Programa de Pós-Graduação em Odontologia, Universidade Estadual da Paraíba (UEPB) 58429-500 Campina Grande - PB, Brasil https://orcid.org/0000-0002-6721-0745

Wliana Pontes DE LIMA

Programa de Pós-Graduação em Odontologia, Universidade Estadual da Paraíba (UEPB) 58429-500 Campina Grande - PB, Brasil https://orcid.org/0000-0002-4384-580X

\section{Resumo}

Introdução. Os dentes supranumerários (DS) são definidos como dentes ou qualquer estrutura odontogênica que estão em excesso em determinada região da arcada dentária. Dependendo da localização anatômica, os DS podem ocasionar falhas na erupção, diastemas, reabsorção radicular, deslocamento dos dentes adjacentes, dilaceração e formação de lesões odontogênicas. Nestes casos, o tratamento de escolha é a exodontia do DS. Objetivo. Relatar um caso de exodontia de DS incluso assintomático em região posterior de mandíbula. Relato do caso. Paciente do sexo masculino, 28 anos de idade, foi encaminhado por um ortodontista para realização de exodontia de DS incluso na região dos dentes 33 a 35, identificado por meio de radiografia panorâmica. A tomografia computadorizada de feixe cônico (TCFC) revelou inclinação distal e posição transversal do DS, cuja coroa estava localizada entre os dentes 34 e 35, com raiz próxima ao ápice radicular do dente 33 e proximidade com o forame mentual e com a raiz do dente 34 . Diante da necessidade de tratamento ortodôntico, realizou-se a exodontia do referido dente. O paciente retornou após oito dias para remoção das suturas, sem relatar queixas. Decorridos 60 dias após a cirurgia, o paciente não referiu qualquer sintomatologia na região onde 0 DS havia sido extraído. Conclusão. O DS é uma das anomalias de desenvolvimento mais comuns em humanos. Ressalta-se a importância dos exames imaginológicos para a identificação e localização precisa deste tipo de anomalia, sendo essencial no caso ora relatado, em que esses exames foram essenciais para o diagnóstico e correto planejamento cirúrgico.

Descritores: Dente Supranumerário; Tomografia Computadorizada de Feixe Cônico; Cirurgia Bucal.

\section{Abstract}

Introduction. Supernumerary teeth are defined as teeth or any odontogenic structure that are in excess in a certain region of the dental arch. Depending on the anatomical location, supernumerary teeth can cause flaws in the eruption, diastema, root resorption, displacement of adjacent teeth, laceration and the formation of odontogenic lesions. In these cases, the treatment of choice is extraction. Objective. To report a case of extraction of supernumerary tooth even asymptomatic in the posterior region of the mandible. Case report. A 28-year-old male patient was referred by an orthodontist to perform supernumerary tooth extraction in the region of teeth 33 to 35 , identified by means of panoramic radiography. Cone beam computed tomography (CBCT) revealed distal inclination and transverse position of the supernumerary tooth, whose crown was located between teeth 34 and 35, with a root close to the root apex of tooth 33 and proximity to the mental foramen and the tooth root 34 . In view of the need for orthodontic treatment, tooth extraction was performed. The patient returned after eight days to remove the sutures, without reporting any complaints. After 60 days after surgery, the patient did not report any symptoms in the region where the supernumerary tooth had been extracted. Conclusion. Supernumerary tooth is one of the most common developmental anomalies in humans. The importance of imaging tests for the identification and precise location of this type of anomaly is emphasized, being essential in the case now reported, in which these tests were essential for the diagnosis and correct surgical planning.

Descriptors: Tooth, Supernumerary; Cone-Beam Computed Tomography; Surgery, Oral.

\section{Resumen}

Introducción. Los dientes supernumerarios (DS) se definen como dientes o cualquier estructura odontogénica que se encuentran en exceso en una determinada región del arco dentario. Dependiendo de la localización anatómica, los DS pueden provocar defectos en la erupción, diastema, reabsorción radicular, desplazamiento de dientes adyacentes, laceración y formación de lesiones odontogénicas. En estos casos, el tratamiento de elección es la extracción del DS. Objetivo. Comunicar un caso de extracción dentaria de DS aún asintomática en la región posterior de la mandíbula. Reporte de un caso. Un paciente masculino de 28 años fue remitido por un ortodoncista para realizar una extracción de DS en la región de los dientes 33 a 35, identificados mediante radiografía panorámica. La tomografía computarizada de haz cónico reveló inclinación distal y posición transversal del DS, cuya corona estaba ubicada entre los dientes 34 y 35, con una raíz cercana al ápice de la raíz del diente 33 y proximidad al agujero mentoniano y la raíz del diente. 34. Ante la necesidad de un tratamiento de ortodoncia, se realizó la extracción del diente. El paciente regresó a los ocho días para retirar las suturas, sin reportar ninguna queja. A los 60 días de la cirugía, el paciente no refirió ningún síntoma en la región donde se había extraído lo DS. Conclusión. EI DS es una de las anomalías del desarrollo más comunes en los seres humanos. Se enfatiza la importancia de las pruebas de imagen para la identificación y localización precisa de este tipo de anomalías, siendo fundamentales en el caso ahora reportado, en el que estas pruebas fueron fundamentales para el diagnóstico y la correcta planificación quirúrgica.

Descriptores: Diente Supernumerario; Tomografía Computarizada de Haz Cónico; Cirugía Bucal.

INTRODUÇÃO

As anomalias dentárias são distúrbios no desenvolvimento ou crescimento dos dentes que podem estar relacionadas tamanho, forma e número ${ }^{1}$. Os dentes supranumerários (DS) fazem parte do grupo de anomalia de número e são definidos como dentes ou qualquer estrutura odontogênica que estão em excesso em determinada região da arcada dentária ${ }^{2,3}$. 
Esses dentes estão além dos 20 decíduos e/ou dos 32 permanentes, erupcionados ou que permaneceram inclusos ${ }^{1-3}$.

Os DS podem ser encontrados em cerca de $1 \%$ da população ${ }^{4}$, acometendo com maior frequência indivíduos do sexo masculino. ${ }^{5-7}$ Sua prevalência varia de $0,1 \%$ a $3,8 \%$ para dentes permanentes $^{6,8,9}$ e de $0,2 \%$ a $1,9 \%$ para dentes decíduos $^{6,8}$. Esses dentes apresentam morfologia variável, podendo se assemelhar a um dente ou possuir anatomia bastante peculiar. De acordo com a morfologia, os DS podem ser classificados em cônico ou tuberculado ${ }^{1,2}$.

Os DS podem ser encontrados unilateralmente ou bilateralmente nos ossos gnáticos, ${ }^{9}$ sendo mais comuns na região anterior de maxila ${ }^{2,6,8}$, especialmente entre os incisivos centrais superiores A maioria dos DS não provoca danos à dentição, sendo descobertos ocasionalmente em radiografias de rotina $^{2}$. Por outro lado, dependendo da localização anatômica, os DS podem ocasionar falhas na erupção, diastemas, reabsorção radicular, deslocamento dos dentes adjacentes, dilaceração e formação de lesões odontogênicas ${ }^{7,10}$. Nestes casos, o tratamento de escolha é a exodontia do DS ${ }^{1,7,10}$. Diante do exposto, o objetivo do presente estudo é relatar um caso de exodontia de DS incluso assintomático em região posterior de mandíbula.

CASO CLÍNICO

Paciente do sexo masculino, 28 anos de idade, foi encaminhado por um ortodontista para realização de exodontia de DS identificado em radiografia panorâmica. Nos exames físicos extra e intraoral não foram observadas alterações. A radiografia panorâmica revelou um DS incluso na região dos dentes 33 a 35 (Figura 1).

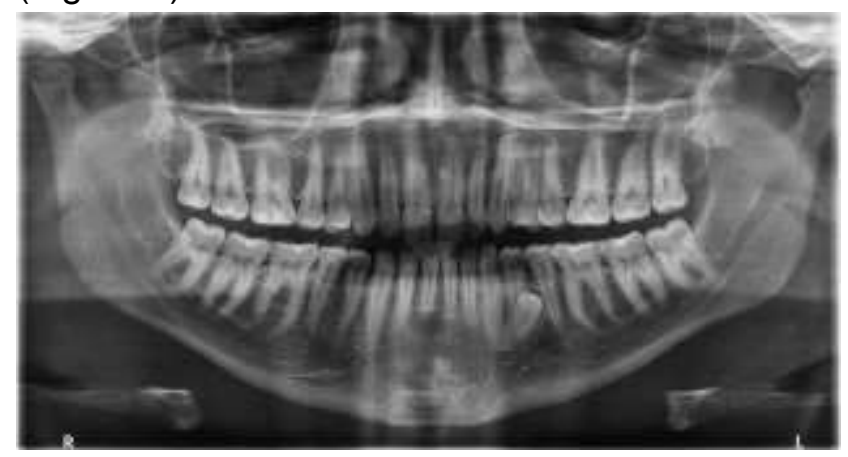

Figura 1: Radiografia panorâmica evidenciando dente supranumerário entre os dentes 33 e 35.

Dessa forma, para se obter uma visualização tridimensional da região foi solicitada tomografia computadorizada de feixe cônico (TCFC), a qual revelou inclinação distal e posição transversal do DS. A coroa do supranumerário estava localizada entre os dentes 34 e 35, com raiz próxima ao ápice radicular do dente 33 (Figura 2). Notou-se também dilaceração radicular no terço apical, e proximidade com o forame mentual e com a raiz do dente 34 .

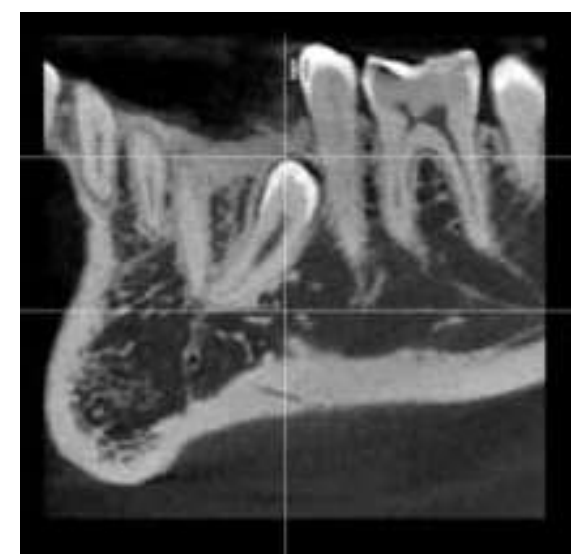

Figura 2:TCFC, em corte coronal, revelando proximidade da raiz do dente supranumerário com o ápice radicular do dente 33.

O paciente foi informado sobre os riscos cirúrgicos inerentes ao procedimento e concordou com a realização da cirurgia. No préoperatório realizou-se antissepsia extraoral com digluconato de clorexidina a 2\% (Riohex, Rioquímica Ltda, Goiânia, Brasil) e bochecho intraoral com digluconato de clorexidina a $0,12 \%$ (Perioplak, reymer Ltda, Goiânia, Brasil). Posteriormente, a região foi anestesiada com mepivacanína a 2\% com epinefrina 1:100.000 (Mepiadre, Nova DFL, Rio de janeiro, Brasil) para bloqueio do nervo mentoniano e infiltrativas locais próximas à região de assoalho, dos dentes 32 a 36 . Após a anestesia, foi realizada incisão intrasulcular, por lingual, da região do dente 32 ao 36 , deslocando o retalho com uma cureta de Molt. Em seguida, foi realizada osteotomia para exposição do DS, utilizando broca esférica multilaminada $\mathrm{n}^{\circ} 6 \mathrm{em}$ alta rotação e irrigação simultânea com soro fisiológico a 0,9\% (Figura 3).

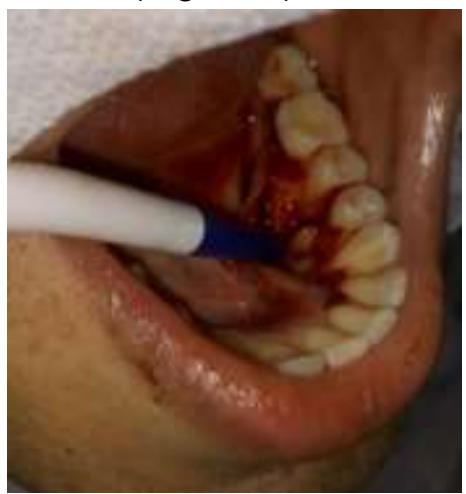

Figura 3: Fotografia mostrando exposição da coroa do dente supranumerário para posterior odontosecção.

Logo após, foi realizada a odontosecção, separando a coroa da raiz, com auxílio da broca cirúrgica tronco-cônica multilaminada (Zekrya, FG Maillefer, Dentsply Sirona), em alta rotação. 
Para remoção do DS, empregou-se alavanca tipo Seldin curva. Os resíduos foram removidos irrigando-se o local com soro fisiológico. Por conseguinte, houve regularização das bordas da loja óssea com lima para osso. Por fim, o retalho foi reposicionado $e$ as suturas realizadas, com pontos simples (Figura 4).

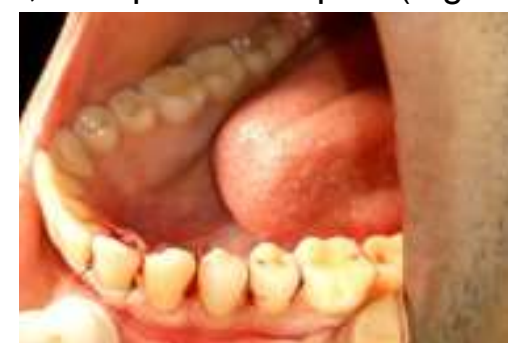

Figura 4: Pontos simples feitos para fechamento do retalho.

Foram dadas recomendações pósoperatórias e prescritos antibiótico (Amoxicilina $500 \mathrm{mg}$ ), a cada 8 horas, por sete dias, e antiinflamatório (Nimesulida $100 \mathrm{mg}$ ), a cada 12 horas, por três dias. O paciente retornou após oito dias para remoção das suturas, sem relatar queixas. Decorridos 60 dias após a cirurgia, o paciente não referiu qualquer sintomatologia na região onde o DS havia sido extraído.

DISCUSSÃO

A presença de DS estar muitas vezes relacionada a síndromes, como a de Gardner, síndrome de Nance-Horan e disostose cleidocraniana $^{11 .}$ Apesar de os DS estarem ligados à síndromes, sua etiologia exata ainda não é conhecida e clara ${ }^{10}$. Existem algumas hipóteses sobre o seu provável mecanismo de formação, englobando fatores genéticos e ambientais, como a hiperatividade da lâmina dentária ${ }^{7,10,12,13}$.

Os exames radiográficos desempenham um papel relevante na identificação e localização do DS, principalmente quando estes encontram-se impactados ou necessitam de intervenção cirúrgica ${ }^{14}$. No caso relatado foi verificada a presença de um DS incluso por meio de radiografia panorâmica, solicitada para fins ortodônticos, evidenciando a importância da radiografia para a localização desta anomalia. Posteriormente, foi solicitada uma TCFC, com o objetivo te ter uma visão tridimensional da região e, por ser considerada o padrão ouro para avaliação de tecidos duros ${ }^{9,10}$. A TCFC auxilia no planejamento cirúrgico, reduzindo possíveis falhas ${ }^{14}$.

O DS pode estar associado a diferentes complicações clínicas, resultando em diastema da linha média, má oclusão devido a espaço insuficiente, dilaceração, atraso ou falha na erupção dentária, problemas periodontais e cárie dentária. Tais complicações podem ter relação com a anatomia e localização do
$\mathrm{DS}^{1,10,14}$. No caso relatado no presente estudo notou-se também uma dilaceração radicular no terço apical, proximidade com o forame mentual e a raiz do dente 34 (terço médio, face lingual) e alteração do contorno, sugerindo reabsorção externa incipiente.

No estudo de Park et al. ${ }^{7}$ realizado a partir da avaliação de prontuários e radiografias de pacientes submetidos a exodontias de DS, identificou-se que a forma cônica foi a mais comumente observada, representando $68 \%$ dos DS. Por outro lado, o formato tuberculado teve incidência de $16 \%$ dos DS, apesar da menor incidência esteve associado a $69 \%$ das complicações.

Existem diferentes opções de tratamento para pacientes com presença de DS. Nos casos assintomáticos, sem evidências radiográficas de lesões e ausência de interferência na movimentação ortodôntica pode haver monitoração por meio de radiografias periódicas ${ }^{9}$. Porém, no caso descrito nesse trabalho, o paciente além de apresentar dilaceração radicular no terço apical e indicativo de reabsorção externa incipiente, iria ser submetido ao tratamento ortodôntico. Provavelmente a movimentação decorrente do tratamento causaria danos aos dentes adjacentes. Dessa forma, a exodontia foi 0 manejo adequado para esse caso ${ }^{9}$.

CONCLUSÃO

O DS é uma das anomalias de desenvolvimento mais comuns em humanos. Podem se apresentar assintomáticos, mas também, acarretar danos ao paciente. Ressaltase a importância dos exames imaginológicos para a identificação e localização precisa deste tipo de anomalia, sendo essencial no caso ora relatado, em que esses exames foram essenciais para 0 diagnóstico e correto planejamento cirúrgico. Vale salientar, a importância do diagnóstico realizado de forma precoce, que evita possíveis complicações clínicas bem como favorecer 0 manejo adequado pelo cirurgião dentista.

REFERÊNCIAS

1. Gurler G, Delilbasi C, Delilbasi E. Investigation of impacted supernumerary teeth: a cone beam computed tomograph (cbct) study. J Istanb Univ Fac Dent. 2017;51(3):18-24.

2. Lu X, Yu F, Liu J, Cai W, Zhao Y, Zhao S, et al. The epidemiology of supernumerary teeth and the associated molecular mechanism. Organogenesis. 2017;13(3):71-82.

3. Manchada N, Anthonappa R, King N. Supernumerary teeth formation following subluxation of primary incisors. Dental Traumatol. 2019;35:212-15. 
4. Costa SM, de Jesus AO, Silveira RL, Amaral MBF. Supernumerary nasal tooth removed with a modified maxillary vestibular approach: case report and literature review. Oral Maxillofac Surg. 2019;23(2):247-52.

5. Cammarata-Scalisi F, Avendaño A, Callea M. Main genetic entities associated with supernumerary teeth. Arch Argent Pediatr. 2018;116(6):437-44.

6. Kiso H, Takahashi K, Mishima S, MurashimaSuginami A, Kakeno A, Yamazaki T, et al. Third dentition is the main cause of premolar supernumerary tooth formation. J Dent Res. 2019;98(9):968-74.

7. Park SY, Jang HJ, Hwang DS, Kim YD, Shin $\mathrm{SH}$, Kim UK, et al. Complications associated with specific characteristics of supernumerary teeth. Oral Surg Oral Med Oral Pathol Oral Radiol. 2020;130(2):150-55.

8. Arikan V, Cumaogullari O, Ozgul BM, Oz FT. Investigation of SOSTDC1 gene in nonsyndromic patients with supernumerary teeth. Med Oral Patol Oral Cir Bucal. 2018;23(5):e531-39.

9. Belmehdi A, Bahbah S, El Harti K, El Wady W. Non syndromic supernumerary teeth: management of two clinical cases. Pan Afr Med J. 2018;29:163.

10.Zhao L, Liu S, Zhang R, Yang R, Zhang K, Xie $X$. Analysis of the distribution of supernumerary teeth and the characteristics of mesiodens in Bengbu, China: a retrospective study. Oral Radiol. 2021;37(2):218-23.

11. Ma D, Wang X, Guo J, Zhang J, Cai $T$. Identification of a novel mutation of RUNX2 in a family with supernumerary teeth and craniofacial dysplasia by whole-exome sequencing. A case report and literature review. Medicine (Baltimore). 2018;97(32):e11328.

12. Haghanifar S, Moudi E, Abesi F, Kheirkhah F, Arbabzadegan N, Bijani A. Radiographic evaluation of dental anomaly prevalence in a selected Iranian population. J Dent (Shiraz). 2019;20(2):90-4.

13. Scully $A$, Zhang $H$, Kim-Berman $H$, Benavides E, Hardy NC, Hu JC. Management of two cases of supernumerary teeth. Pediatr Dent. 2020; 42(1):58-61.

14. Mahto RK, Dixit S, Kafle D, Agarwal A, Bornstein M, Dulal S. Nonsyndromic bilateral posterior maxillary supernumerary teeth: a report of two cases and review. Case Rep Dent. 2018;2018:5014179.

\section{CONFLITO DE INTERESSES}

Os autores declaram não haver conflitos de interesse

\section{AUTOR PARA CORRESPONDÊNCIA}

\section{Wliana Pontes de Lima}

Programa de Pós-Graduação em Odontologia, Universidade Estadual da Paraíba (UEPB)

Rua Baraúnas, 351 - Bairro Universitário 58429-500 Campina Grande - PB, Brasil

E-mail:wli_pontes@outlook.com 